\title{
Platelet Function Disorders in Adolescents with Heavy Menstrual Bleeding: Clinical Presentations, Laboratory Testing and Treatment Options
}

Lawrence S Amesse $^{1 *}$, James A French ${ }^{2}$ and Teresa Pfaff-Amesse ${ }^{1}$

${ }^{1}$ Section of Pediatric/Adolescent Gynecology, Atlantic Reproductive Associates, Boynton Beach, Florida, USA

${ }^{2}$ Division of Hematology and Oncology, Department of Pediatrics, Dayton Children's Hospital, Dayton, Ohio, USA

\begin{abstract}
Background: Platelet Function Disorders (PFD) is receiving increasing attention as an important etiology of Heavy Menstrual Bleeding (HMB) in adolescents. An understanding of presentation profiles, diagnostic limitations and effective treatment regimens for these disorders are important for effective management of these disorders.

Methods: This study reviews the literature on the diagnosis and treatment of platelet function disorders in adolescents with heavy menstrual bleeding.

Results: Heavy menstrual bleeding affects the quality of life of many adolescents, and PFDs appearing to be an important underlying etiology of this disorder. The storage pool subtype is the most prevalent of the platelet functional defects in these patients. Standard platelet functions screening has a number of limitations in diagnosing many of the PFD subtypes, and platelet aggregation studies and electron microscopy are important diagnostic adjuncts. Hormonal and non-hormonal treatment regimens are available and treatment recommendations will depend on the severity of bleeding.

Conclusion: Clinicians treating adolescent with HMB should be aware of PFDs and the diagnostic limitations in detecting many of the subtypes. Management depends on the severity of bleeding.
\end{abstract}

Keywords: Heavy menstrual bleeding; Platelet function disorders; Hypothalamic pituitary ovarian

\section{Introduction}

Heavy menstrual bleeding (HMB) is a common problem in adolescent girls where it comprises a significant public health problem [1]. HMB affects almost one-third of adolescent girls at the time of the menarche. Reports from multiple studies indicate that between 5 and 10 percent of young girls in this age group will seek medical treatment and of those, approximately one-half will experience surgical intervention [1-3].

For young girls that do not seek medical assistance, there is a high probability that HMB will affect their daily lives. Indeed, HMB has been reported to negatively impact several quality of life parameters. Often these girls will miss school, sports and other social activities because of associated symptoms and there are psychosocial factors that deserve consideration. Hygiene issues are important factors, particularly in developmentally delayed adolescents. The long, heavy periods are often combined with additional symptoms including dysmenorrhea and pelvic pain and are usually accompanied by anemia with its associated symptoms of easy fatigue and energy loss, headaches and light-headedness. Reasons for not initiating medical consultation are unclear. Directed review of family history often reveals a family remarkable for heavy periods in several relatives and HMB is often considered by the patient and her mother to be "normal" for the family (Figure 1) [4].

A common cause of $\mathrm{HMB}$ in adolescents is immaturity of the hypothalamic pituitary ovarian (HPO) axis with resultant anovulation. Anovulation is associated with an endometrium proliferationin the absence of luteal-phase progesterone support of the proliferating endometrial glands. The proliferating endometrial glands outgrow their vascular supply with consequent steady shedding of the endometrium, which manifests clinically as heavy, continuous menstrual bleeding. Some experts estimate that immaturity of the HPO axis is the underlying cause of HMB in approximately $75 \%$ of cases in young females [5]. However, HPO axis is a diagnosis of exclusion. The astute clinician should consider other etiologies before assigning HPO axis as the diagnosis as other causes, amenable to treatment, may be

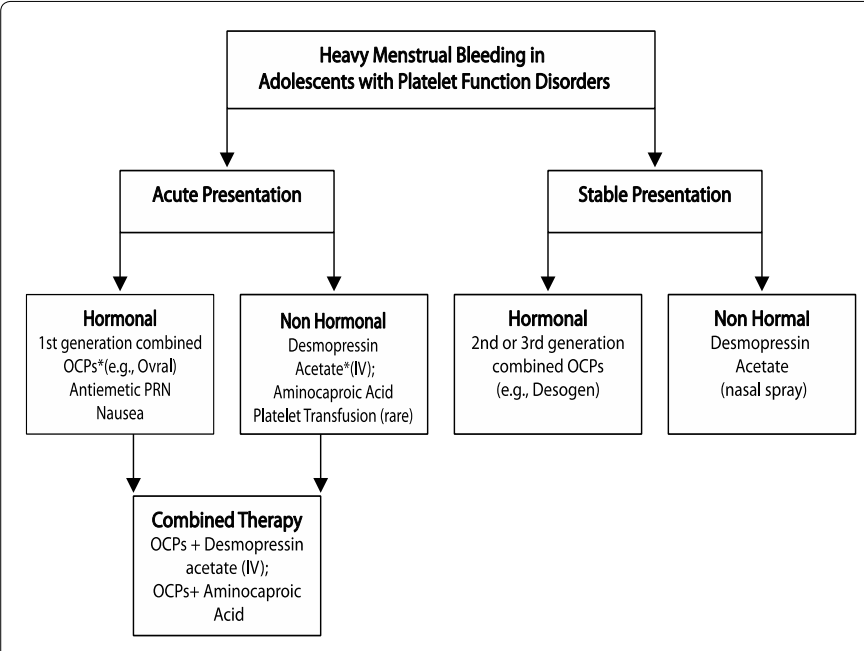

OCPs (oral contraceptive polls); Desmopressin Acetate (1-desamino-8-D-arginine vasopressin)

Figure 1: Flow Diagram of Treatment Strategies for Heavy Menstrual Bleeding.

*Corresponding author: Lawrence S Amesse, Atlantic Reproductive Associates, 10301 Hagen Ranch Road, Suite 6, Boynton Beach, Florida 33437 USA, Tel: 937-545-2011; Fax: 937-458-5005; E-mail: Isamesse@gmail.com

Received September 24, 2013; Accepted December 24, 2013; Published December 29, 2013

Citation: Amesse LS, French JA, Pfaff-Amesse T (2013) Platelet Function Disorders in Adolescents with Heavy Menstrual Bleeding: Clinical Presentations, Laboratory Testing and Treatment Options. J Blood Disorders Transf 5: 186. doi: 10.4172/2155-9864.1000186

Copyright: (C) 2013 Amesse LS, et al. This is an open-access article distributed under the terms of the Creative Commons Attribution License, which permits unrestricted use, distribution, and reproduction in any medium, provided the original author and source are credited. 
Citation: Amesse LS, French JA, Pfaff-Amesse T (2013) Platelet Function Disorders in Adolescents with Heavy Menstrual Bleeding: Clinical Presentations, Laboratory Testing and Treatment Options. J Blood Disorders Transf 5: 186. doi: 10.4172/2155-9864.1000186

Page 2 of 5

overlooked. Indeed, HMB is often the only indication of an underlying endocrine or bleeding disorder. Structural problems such as fibroids or polyps are not as likely considerations in this age group although congenital anomalies or foreign bodies are sometimes seen and should also be considered in the evaluation. An ultrasound of the pelvis should be considered to exclude these disorders.

Endocrine disorders such as thyroid abnormalities should be considered as both hyper-and hypothyroid conditions can be associated with abnormal uterine bleeding and can be corrected. In general, thyroid disorders can be excluded by obtaining TSH levels [6].

Coagulopathies should always be considered and testing should be initiated particularly if there is significant anemia. Many clinicians will consider screening laboratory studies for a von Wille brand Disease (VWD) diagnosis and will obtain a von Wille brand Factor (VWF) assay and ristocetin cofactor activity levels. In the presence of significant anemia, other studies including measuring Factor VIII levels and VWF multimers analysis are often included. Other coagulopathies and bleeding disorders, including platelet function defects, often go unrecognized unless the clinician is aware of their role in excessive menstruation.

Other Factors: Although not as common in this age group, structural problems such as fibroids, endometrial polyps, adenomyosis and malignancy as well as foreign bodies need to be considered as a cause if heavy menstrual bleeding. They may be confounding factors in the evaluation process.

\section{Platelet Function Disorders}

\section{Background}

Platelet function disorders (PFD) are now thought to be a significant cause of heavy menstrual periods in adolescents. Unlike VWD, these disorders represent a large heterogenous group of platelet functional defects for which cost effective testing is not as easily demonstrated. Recent studies, however have indicated that they are as prevalent as VWD in teenagers with heavy menstruation $[4,7,8]$. This review will concentrate on platelet function disorders; their natural history in adolescents with heavy menstrual periods and will provided suggested testing and treatment regimens.

\section{Types of platelet function disorders}

Platelet function disorders can be either inherited or acquired and encompass either qualitative or quantitative platelet abnormalities. Qualitative defects reflect alterations in platelet function: aggregation, adhesion or secretion, where as quantitative abnormalities are composed of alterations in platelets numbers, such as those encountered in thrombocythemia, thrombocytosis, and thrombocytopenia (Table 1) $[9,10]$. Depending on the type of abnormality they can result in either thrombosis or bleeding tendencies. The most common congenital PFDs are secretion defects, and the Storage Pool (SP) subtype is the most frequently identified [9]. One recent study reported that $64 \%$ of adolescents with platelet-associated HMB had the SP defect, which was definitively identified using electron microscopy studies of platelets [11].

The SP defects reflect altered number or contents of the platelet granules, Figure 2 . The defects are seen in platelet dense $(\delta)$-granules, $\alpha$-granules or a combination of both the $\alpha \delta$-granules [9]. Dense $(\delta)$ granule deficiency is the most common and not unexpectedly is the most common PFD type seen in teenagers with platelet dysfunctionassociated HMB $[9,11]$. Although $\delta$-granule deficiencies are found in individuals unrelated to specific syndromes, it is important to note that they can be associated with rare syndromes such as Chediak-Higashi and Hermansky-Pudlak and clinical correlation may be indicated [12].

\section{Diagnostic Evaluation}

\section{Presentation Profile}

Adolescents with heavy menstrual bleeding should be carefully evaluated and the presentation profile is quite important when one is considering a platelet function disorder etiology. Most young adolescents with inherited bleeding disorders, particularly those with VWD, PFDs and other coagulation factor deficiencies, clinically manifest $\mathrm{HMB}$ at the onset of the menarche [9]. Menstruation represents a major hemostatic challenge and at menarche, pre adolescent and teenage girls with these disorders will often be seen in the emergency room with complaints of acute onset of heavy vaginal bleeding and significant anemia with hematocrits in the $20 \%$ and hemoglobin levels, 4-5 mg/dL [11,12]. Often fluid resuscitation including several blood transfusions is required to replace acute blood loss. To treat ongoing bleeding, it is often necessary to implement appropriate pharmacologic therapeutics. The most common presentation scenario in patients with platelet dysfunction-associated $\mathrm{HMB}$, however, is a teenager presenting to the clinician's office with complaints of chronic heavy

\begin{tabular}{|l|l|}
\hline Defect & Disorder \\
\hline Adhesion & $\begin{array}{l}\text { Bernard-Soulier syndrome } \\
\text { von Willebrand disease, platelet-type }\end{array}$ \\
\hline Aggregation & $\begin{array}{l}\text { Glanzmann's thrombasthenia } \\
\text { Congenital afibrinogenemia }\end{array}$ \\
\hline Cytoskeleton & $\begin{array}{l}\text { Wiscott-Aldrich syndrome } \\
\text { MYH9-related disorders }(18)\end{array}$ \\
\hline Membrane phospholipids & Scott syndrome \\
\hline Secretion and: & $\begin{array}{l}\text { Storage pool deficiency }(\alpha, \delta, \alpha \delta \text { granules) } \\
\text { Quebec platelet disorder }(\alpha \text { granules) }\end{array}$ \\
\hline Granule anomalies & $\begin{array}{l}\text { Platelet receptor defects } \\
\text { G-protein activation defects } \\
\text { Phosphatidylinositol metabolism defects } \\
\text { Calcium mobilization defects } \\
\text { Protein Phosphorylation defects } \\
\text { Arachadonic acid/thromboxane A2 synthesis defects }\end{array}$ \\
\hline
\end{tabular}

Table 1: Qualitative Platelet Function Disorders. *

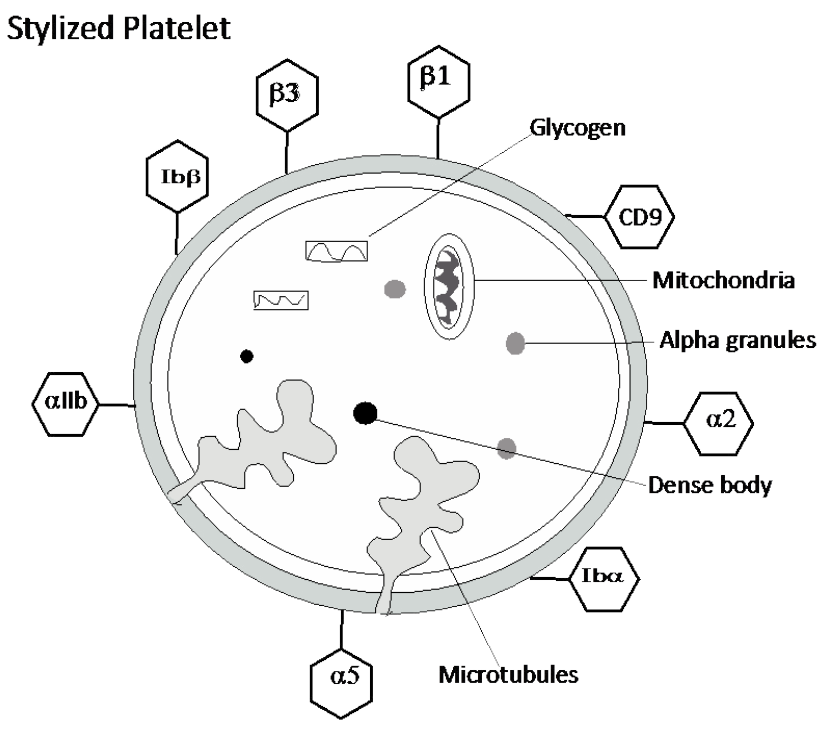

Figure 2: Stylized Platelet. 
menstrual periods and signs of mild anemia. A concern for physicians caring for patients with more subtle presentations is when to initiate a full diagnostic evaluation. Hematocrits in these girls are often in the low normal ranges, and since the patient is inexperienced with normal menstrual blood flow, quantitation of blood loss can be difficult to assess.

\section{Bleeding History}

PFD disorders are difficult to recognize in adolescents with abnormal uterine bleeding, and a clinician will need a strong index of suspicion in order to initiate directed questioning and ordering laboratory testing [12]. Initial questioning should be directed towards defining the patient's own history of bleeding tendencies as well as that of the family members. Questioning should be directed towards discovery of common indicators of an underlying bleeding disorder such as easy bruising (i.e., bruises $>5 \mathrm{~cm}$ diameter occurring $\geq 1 /$ month duration), epistaxis that occurs $\geq 1$ /month duration and gingival or other mucosal bleeding as well as excessive bleeding associated with surgery, dental procedures and minor trauma. It is particularly important to obtain a family history of these same problems, and to ask about ancestors with heavy menstrual bleeding $[11,12]$. It is sometimes possible to distinguish PFDs as well as other bleeding diatheses, i.e., VWD, factor deficiencies, by determining the onset of bleeding after a hemostatic challenge $[11,12]$. A majority of adolescents with underlying bleeding diatheses have heavy menstrual bleeding within the first year of menarche and so is important to determine when the heavy bleeding was first noticed.

A thorough menstrual history is essential in these patients, and it is important to determine the number of bleeding days in a typical menstrual cycle. Despite missing several school days and other activities, this adolescent subgroup often consider their long (>7days) or heavy menses to be "normal" in their family. Often the patient and her mother will indicate that the bleeding is similar to that experienced by the mother herself as well as the mother's sisters, mother, aunts and even paternal family members. Many patients will have relatives that had early hysterectomies because of long and/or heavy menstrual bleeding. Family histories of bleeding tendencies can also assist in identifying PFDs [11].

\section{Signs and Symptoms}

The patient will often complain about headache, dizziness and easy fatigue with diminished energy. The examining physician should look for signs of anemia including tachycardia, pale skin and mucosa and decreased blood pressure, in addition to the presence of petechiae, ecchymosis and purpura. In many cases a pelvic examination is difficult in these young adolescents. However an external examination to approximate blood loss as well as a trans-abdominal ultrasound of the pelvis to exclude any uterine abnormalities as well as presence of foreign bodies can replace an internal pelvic examination, particularly in non-sexually active teens.

\section{Laboratory Testing}

There are no standard, definitive studies for screening or diagnosing a PFD and this is attributed to their heterogeneity. In addition, there is wide variation in testing costs. Another consideration clinicians should be aware of is the limitations in standardized testing with different laboratories showing marked variation in platelet function assays results. Furthermore, standard screening tests lack sensitivity for detecting many of the variations seen in PFDs. In some cases, the diagnostic test will detect a PFD missed previously by screening studies
[11]. Although protocols exist for obtaining, processing and analyzing blood samples, interpretation, and specific diagnostic guidelines for these disorders are lacking [9].

The stated limitations aside, obtaining a complete blood count with platelet counts along with assessment of platelet size and morphology is traditionally the first approach, and will aid the clinician in excluding any associated syndromes. Preliminary screening studies are aimed at detecting coagulopathies. Both PT and a PTT are considered a standard part of initial testing. Ristocetin cofactor (VWF:RCo), VWF antigen (VWF:Ag), VWF:RCo:VWF ratios and Factor VIII (FVIII:C) activity levels are performed to detect VWD. Measuring $\beta$-hCG levels is indicated to exclude pregnancy in any teen presenting with heavy uterine bleeding. When the stated testing results are within normal ranges, platelet function testing is indicated. Indeed, quantitative platelet defects are ruled out in the presence of normal platelet counts.

The cost effective next step of the evaluation is to perform platelet function screening. Results of the platelet testing can be influenced by interfering drugs, including SSRI's, tricyclic antidepressants, prostaglandin inhibitors, and some antibiotics [11]. Most laboratories recommend discontinuing any of these medications for at least 7-10 days prior to testing.

Closure time (CT) testing using the platelet function analyzer (PFA) system is a screening tool for platelet dysfunction abnormalities that has nearly replaced template bleeding times (BT) at most centers. However, various studies indicate the PFA system lacks both sensitivity and specificity for detecting PFDs and thus, is of limited utility $[11,12]$.

Prolonged (abnormal) closure times using the PFA system are followed by testing algorithms that recommend confirmatory studies such as platelet aggregation assays. Correspondingly, when closure time results with the PFA system are normal, it is up to the clinician to determine whether additional testing is indicated. The decision to order additional testing is often based on clinical index of suspicion and family histories remarkable for bleeding tendencies. In approximately $20 \%$ cases, platelet aggregation assays can detect the presence of a PFD despite normal CTs with the PFA system as a screening test $[11,12]$.

Platelet aggregation assays are widely available and are useful in detecting PFDs. However, this modality has been reported give normal testing results a number of patients subsequently diagnosed with platelet $\delta$-granule deficiencies by Electron Microscopy (EM) and other definitive testing $[11,13]$. EM can accurately identify platelet $\delta$-granule deficiencies and other ultrastructural abnormalities. Therefore, it should be considered in cases in which there is a high clinical suspicion of an underlying inherited bleeding disorder and/or a family history of bleeding tendencies, particularly after other causes have been excluded [8]. Since the testing is not widely available, platelet samples may have to be referred to specialized centers that routinely offer this study.

Because of the heterogenous nature of these disorders correct diagnosis will often incorporate multiple testing modalities not traditionally used in may diagnostic laboratories.

One of this is flow cytometry analysis of GP-1b-IX-V, GPIIb/IIIa, GPVI which are unique in certain platelet function disorders. Another testing modality is thromboelastography. This assay measures the efficiency of clot formation as well as the viscoelastic properties of the clot. The assay has a number of modifications but essentially uses a small curvette that is slowly rotated to imitate sluggish venous blood flow. Using a thin wire, a clot begins to form between the probe and the side of the cuvette, and the strength and thickness of the clot is measured over time [14] 
Citation: Amesse LS, French JA, Pfaff-Amesse T (2013) Platelet Function Disorders in Adolescents with Heavy Menstrual Bleeding: Clinical Presentations, Laboratory Testing and Treatment Options. J Blood Disorders Transf 5: 186. doi: 10.4172/2155-9864.1000186

Page 4 of 5

\section{Treatment}

Options for treating PFDs will depend on the severity of the bleeding. Recommended modalities, summarized in Table 2 [15], include hormonal, non-hormonal and combined approaches.

Acute vaginal bleeding with significant blood loss requires empirical treatment started immediately, before the patient's laboratory tests are available for interpretation. One approach is to follow treatment regimens designed for patients with VWD and HMB. The treatment modality contains high doses of estrogen. It is important to draw blood for VWF assays, other coagulation testing and platelet function studies prior to initiating any therapy as treatment protocols can be modified after factor deficiency and platelet function study results are obtained.

\section{Hormonal}

A number of hormonal treatment options to control bleeding in patients with PFD are available, and most include the use of high dose estrogens. The exact mechanism through which high dose estrogens promote hemostasis is not completely understood. It is thought that elevated circulating estrogens increase clotting factors and stimulate platelet aggregation that in turn assists in achieving hemostasis. Oneregime involves IV estrogens such as Premarin ${ }^{\circledR}$ given in doses of $25 \mathrm{mg}$ every 4-6 hours [16]. When the bleeding stops, an oral form of estrogen combined with progestin is administered. Combined estrogen and progestin are often given in the form of a combined first generation oral contraceptive pill.

Another strategy is to begin with combined oral contraception pills (OCPs) in place of IV estrogen, and this is particularly useful in patients who do not have nausea and vomiting. First generation OCPs such as $\mathrm{Ovral}^{\mathbb{B}}$ (50 $\mu \mathrm{g}$ ethinyl estradiol and $0.5 \mathrm{mg}$ of norgestrel) are particularly appropriate, and they are administered as a single tablet every 4-6 hours. The dose is incrementally decreased when the bleeding stops, and this is usually $\sim 24$ hours after commencing treatment.

In some cases non-hormonal therapy, discussed in the next section, can be combined with the high dose estrogen regimen to control the initial bleeding. In addition, an anti-emetic such as ondansetron $\left(\right.$ Zofran $^{\circledR}$ ) should be considered to control nausea associated with high doses of estrogen.

Protocols to taper the amount of estrogen will vary and involve gradually increasing the interval between OCP dosing. One protocol involves giving the patient a first generation OCP every eight hours for two days and then every 12 hours for three days and finally one OCP a day. The patient may be continued on that regimen for a number of months skipping the hormone free OCPs so as to avoid menstrual flow. If the patient is well controlled on this regimen, a second or third generation OCP can be substituted and the patient allowed menstruating by continuing with the hormone free interval pills. Some patients with PFDs will have intra-menstrual bleeding on the lower dose OCPs and will need to continue to continue on a higher dose birth control pill.

Patients presenting with only moderate bleeding without anemia can be effectively treated with second or third generation low dose OCPs such as Desogen ${ }^{\circledR}$ [16]. In most cases the patient should be started on once-a-day dosing. These regimens are preferable as they lack the side effects associated with the higher dose first generation OCPs. However, a higher dose OCP may be considered in some cases.

Intrauterine devices (IUD): The progestin-containing intrauterine device, Minera ${ }^{\circledR}$ is an accepted strategy for controlling excessive menstrual bleeding in teens with PFDs. The IUD contains a comparatively strong progestin that contributes to endometrial atrophy and reduces menstrual flow. Previous sexually transmitted diseases (STDs) are no longer a contraindication to IUDs. However, adolescents with a history of STDs should be carefully scrutinized and followed appropriately. The copper T IUD is associated with increased menstrual flow and is not recommended for treating heavy menstrual periods.

\section{Non-hormonal}

Non-hormonal treatment is often preferred in some patients and there are a variety of treatment options. One option is $\operatorname{DDAVP}^{\circledR}(1-$ diamino-8-D-arginine vasopressin or Stimate $\left.{ }^{\circledR}\right)$. DDAVP works by releasing von Willebrand antigen and Factor VIII from platelets and endothelial cells. It also increases the expression of glycoprotein $1 \mathrm{~b} /$ IX and CD62 (P-selectin) on platelet cell surfaces, which lead to increased platelet activation and adhesion [17]. One treatment regimen that is used with acute blood loss is to infuse $0.3 \mu \mathrm{g} / \mathrm{kg}$ demsopressin $\left(\right.$ DDAVP $\left.^{\circledR}\right)$ in $30 \mathrm{ml}$ of saline over 30 minute duration. Another regimen used for stable patients is to give DDAVP (Stimate ${ }^{\circledR}$ ) in the form of a nasal spray with one squirt $(150 \mu \mathrm{g})$ per nostril in patients that weigh less than $50 \mathrm{~kg}$, and one squirt to both nostrils for those weighing $50 \mathrm{~kg}$ or more.

Fibrinolysis inhibitor: Aminocaproic acid $\left(\operatorname{Amicar}^{\circledR}\right)$ is another

\begin{tabular}{|c|c|}
\hline Therapeutic Modality & Dosage/Notes \\
\hline $\begin{array}{l}\text { Hormonal } \\
\text { Acute presentation } \\
\left.1^{\text {st }} \text { generation, combined OCP (e.g. Ovral }{ }^{\circledR}\right) \text { Anti-emetic (e.g. } \\
\text { Zofran®) PRN Nausea } \\
\text { Stable presentation } \\
2^{\text {nd }} \text { or } 3^{\text {rd }} \text { generation OCPs } \\
\left.\text { Intrauterine Devices (IUD) (Minera }{ }^{\circledR}\right)\end{array}$ & $\begin{array}{l}\text { One tab Ovral }{ }^{\circledR} \mathrm{PO} q \text { q } \mathrm{hrs} \text { until bleeding stops, then } \downarrow \text { dose to one tab q8hr } \times 3 \text { days, then one tab BID X } \\
2 \text { days, then } 1 \text { tab each day } \\
\text { One tab PO q } 12 \mathrm{hrs} \text { until bleeding diminishes/stops, then continue one tab daily } \\
\text { IUD can now be used in adolescents even with history of Pelvic Inflammatory Diseases. The CuT IUD } \\
\text { is not recommended to control Abnormal Uterine Bleeding }\end{array}$ \\
\hline $\begin{array}{l}\text { Non-hormonal } \\
\text { Acute presentation } \\
\text { Desmopressin }\left(D^{\circledR} D P^{\circledR}\right) \\
\text { Aminocaproic acid }\left(\text { Amicar }^{\circledR}\right) \\
\text { Platelet Transfusion } \\
\text { Stable presentation } \\
\text { DDAVP nasal spray (Stimate }{ }^{\circledR} \text { ) }\end{array}$ & $\begin{array}{l}0.3 \delta \mathrm{g} / \mathrm{kg} \text { DDAVP }{ }^{\circledR} \mathrm{IV} \text { in } 30 \mathrm{ml} \text { of saline in } 30 \mathrm{~min} \text { infusion } \\
100 \mathrm{mg} / \mathrm{kg} \text { Amicar }{ }^{\circledR} \mathrm{IV} \text { or PO followed by } 20 \mathrm{mg} / \mathrm{kg} \text { IV or PO } \mathrm{q} \mathrm{hr} \text { until bleeding is controlled } \\
\text { Reserved for patients w/documented PFDs refractory to pharmacological regimens; pediatric hematolo- } \\
\text { gist consult } \\
1.5 \mathrm{mg} / \mathrm{ml} \text { Stimate } \\
\text { nostrils }\end{array}$ \\
\hline $\begin{array}{l}\text { Combined therapies may be indicated: } \\
\text { Acute presentation } \\
\text { OCP+DDAVP } \\
\text { OCP+Aminocaproic acid }\left(\text { Amicar }^{\circledR}\right)\end{array}$ & $\begin{array}{l}\text { Combined dosages of OCPs \& DDAVP } \\
\text { Combined dosages of OCPs \& Amicar }\end{array}$ \\
\hline
\end{tabular}

Table 2: Strategies for Treating Adolescents with Heavy Menstrual Bleeding due to PFDs*. 
Citation: Amesse LS, French JA, Pfaff-Amesse T (2013) Platelet Function Disorders in Adolescents with Heavy Menstrual Bleeding: Clinical Presentations, Laboratory Testing and Treatment Options. J Blood Disorders Transf 5: 186. doi: 10.4172/2155-9864.1000186

Page 5 of 5

approach to treat platelet-dysfunction associated HMB. A dosage of $100 \mathrm{mg} / \mathrm{kg}$ of the fibrinolysis inhibitor is initially given either IV or PO, followed by $20 \mathrm{mg} / \mathrm{kg}$ IV or PO each hour until control of the bleeding is achieved.

Platelet transfusion: Platelet transfusion is considered a therapy of last resort and is done in consultation with a pediatric hematologist. It is usually administered in patients with documented platelet dysfunction and significant uterine bleeding refractory to other therapeutic approaches. A recent study reports on the generation of functional megakaryocytes and platelets derived from human endometrial stem cells. Consideration of future directions in treatments based on the studies discovery include using generated platelets derived from endometrial stromal stem cells for autologous source of platelet therapy, thus bypassing the usual limitations of transfusion therapy in these patients. Additional studies will be needed to define the feasibly of applying this modality. If successful will offer a novel approach to treating these disorders $[18,19]$.

\section{Conclusions}

Inherited bleeding disorders are an important and common cause of heavy menstrual bleeding in adolescents. VWD-associated HMB is a well known and commonly reported etiology. Platelet dysfunction-associated HMB appears to be more common than previously thought and is an important cause of HMB in young adolescents. However, this disorder may be overlooked by clinicians due to lack of awareness regarding limitations in detecting platelet defects by standard platelet testing. Clinicians treating adolescents with HMB should be aware of these disorders as well as the limitations to current laboratory testing methods and recommended treatment protocols.

\section{Acknowledgement}

Dr. Lawrence Amesse is the recipient of a grant to study platelet function disorders in adolescents with menorrhagia from the CSL Behring Foundation. Drs. Pfaff-Amesse and French were co-investigators.

\section{References}

1. James $\mathrm{AH}$, Ragni MV, Picozzi VJ (2006) Bleeding disorders in premenopausal women: (another) public health crisis for hematology? Hematology Am Soc Hematol Educ Program.

2. Friberg B, Ornö AK, Lindgren A, Lethagen S (2006) Bleeding disorders among young women: a population-based prevalence study. Acta Obstet Gyneco Scand 85: 200-206.
3. Doherty L, Harper A, Russell M (1995) Menorrhagia management options. Ulster Med J 64: 64-71.

4. Jayasinghe $\mathrm{Y}$, Moore $\mathrm{P}$, Donath S, Campbell J, Monagle P, et al. (2005) Bleeding disorders in teenagers presenting with menorrhagia. Aust $\mathrm{N} \mathrm{Z} \mathrm{J}$ Obstet Gynaecol 45: 439-443.

5. Gillani S, Mohammad S (2012) Puberty menorrhagia: causes and management J. Med. Sci 20: 15-18.

6. U.S. Preventive Services Task Force (2004) Guide to Clinical Preventive Services, (2ndedn.), Alexandria, Virginia: International Medical Publishing pp: 1-5.

7. Philipp CS, Dilley A, Miller CH, Evatt B, Baranwal A, et al. (2003) Platelet functional defects in women with unexplained menorrhagia. J Thromb Haemost 1: $477-484$.

8. Mikhail S, Varadarajan R, Kouides $P$ (2007) The prevalence of disorders of haemostasis in adolescents with menorrhagia referred to a haemophilia treatment centre. Haemophilia 13: 627-632.

9. Handin RI (2005) Inherited platelet disorders. Hematology Am Soc Hematol Educ Program.

10. Kunishima S, Saito $H$ (2010)Advances in the understanding of MYH9 disorders. Curr Opin Hematol 17: 405-410.

11. Amesse LS, Pfaff-Amesse T, Gunning WT, Duffy N, French JA 2nd (2013) Clinical and laboratory characteristics of adolescents with platelet function disorders and heavy menstrual bleeding. Exp Hematol Oncol 2: 3.

12. Hayward CP, Rao AK, Cattaneo M (2006) Congenital platelet disorders: overview of their mechanisms, diagnostic evaluation and treatment. Haemophilia 12 Suppl 3: 128-136.

13. Nieuwenhuis HK, Akkerman JW, Sixma JJ (1987) Patients with a prolonged bleeding time and normal aggregation tests may have storage pool deficiency: studies on one hundred six patients. Blood 70: 620-623.

14. Trapani L (2013) Thromboelastography: Current applications, future directions. Open journal of anesthesiology 3: 23-27.

15. (2010) Pediatric Forum. A journal of The Children's Medical Center of Dayton 22.

16. Emans SJ, Laufer M (2012) Goldstein's Pediatric \& Adolescent Gynecology (6th Edn.), Lippincott Williams \& Wilkins, Philadelphia.

17. Leissinger C, Carcao M, Gill JC, Journeycake J, Singleton T, et al. (2013) Desmopressin (DDAVP) in the management of patients with congenital bleeding disorders. Haemophilia .

18. Wang J, Chen S, Zhang C, Stegeman S, Pfaff-Amesse T, et al. (2012) Human endometrial stromal stem cells differentiate into megakaryocytes with the ability to produce functional platelets. PLoS One 7: e44300.

19. Michelson AD, Barnard MR, Krueger LA, Frelinger AL 3rd, Furman MI (2000) Evaluation of platelet function by flow cytometry. Methods 21: 259-270. 